\title{
CDISC SDTM Observational Study Sampling Method Terminology
}

National Cancer Institute

\section{Source}

National Cancer Institute. CDISC SDTM Observational Study Sampling Method

Terminology. NCl Thesaurus. Code C127260.

Terminology associated with the observation study sampling method codelist of the Clinical Data Interchange Standards Consortium (CDISC) Study Data Tabulation Model (SDTM). 